Dhaka Univ. J. Biol. Sci. 20(1): 1-6, 2011 (January)

\title{
FORENSIC MICROSATELLITE TH01 AND MALARIA PREDISPOSITION
}

\author{
Shafiul Alam, Ahmad Ferdous, Md. Eunus Ali, Asma Ahmed, \\ Apala Farhat Naved ${ }^{1}$ AND Sharif AKHTERUZZAMAN* \\ Department of Biochemistry and Molecular Biology, University of Dhaka, \\ Dhaka-1000, Bangladesh
}

Key words: Microsatellites, Allele, Genotype, Locus, Malaria

\begin{abstract}
It is hypothesized that microsatellite locus TH01, which is located in close proximity to beta-globin gene and immune regulatory region in human, plays a role in malaria predisposition. In this study, authors verified the association of microsatelite allele (AATG repeat unit) at TH01 locus with malaria infection. The study provided significant evidence that allele- 9 at this locus in $P$. falciparum infected individual illustrate the genetic predisposition towards the disease. Moreover, the predominance of allele- 9 at TH01 in the individuals inhabiting malaria endemic area suggests that genetic predisposition towards malaria is an archaic phenomenon.
\end{abstract}

\section{Introduction}

Microsatellites are tandemly repeated sequences of DNA, usually 2 - 7 bp in length and spread over the entire human genome.(1) They are highly polymorphic in repeat sequence and length, and occur on an average every 10,000 nucleotides.(2) This broad genomic distribution and high level of diversity led to the application of microsatellites as genetic markers for parentage testing and forensic applications. ${ }^{(3)}$

Plasmodium falciparum malaria is a major cause of morbidity and mortality in many developing countries, especially in sub-Saharan Africa, where childhood mortality is over one million per year.(4) Over the past 50 years accumulated evidence indicate that genetic factors can influence the onset, progression, severity of disease and ultimate outcome of malaria infection in humans. This genetic component is complex, multigenic, and several studies have revealed important interactions between host genome and the malarial parasite. Ethnic differences in disease severity have been reported in endemic areas of West Africa, ${ }^{(5)}$ and segregation analysis has indicated a genetic component in the control of malaria infection.(6) Independent studies have also pointed at an important genetic control of host immune response in malaria.(7) Polymorphism in the coding genes, such as Duffy antigen chemokine receptor (DARC), hemoglobin variants $(\mathrm{HbE}, \mathrm{HbC}$, $\mathrm{HbS}$ ), $\alpha$ - and $\beta$-thalassemia, glucose-6-phosphate dehydrogenase, HLA (HLA-B53, DRB1*1302) and other immune regulatory have been documented with malaria resistance. ${ }^{(8-11)}$

*Corresponding author: <sharif_akhteruzzaman@yahoo.com>. ${ }^{1}$ National Forensic DNA Profiling Laboratory, Dhaka Medical College, Dhaka-1000, Bangladesh. 
Human genome harbors enormous diversity by neutral polymorphisms, such as microsatelite sequences, which are recombinant 'hotspots' owing to their repetitive length sequences. Microssatellite alleles in bacterial species, on the other hand, are known to aid their survival in extreme environmental conditions. Micosatellite sequence present within the Opa genes of prokaryotes, namely Neisseria gonorrhoea and Hemophilus influenzae, aids their survival in fatal environmental condition ${ }^{(12)}$. Therefore, this study explored whether allelic variation in microsatellite region in human genome has any role in survival against environmental stress or infections.

\section{Materials and Methods}

Liquid blood samples were collected from 43 patients clinically positive for Plasmodium falciparum and individuals belonging to three ethnic population, such as Chakma ( $n=109)$, Rakhain $(n=85)$ and Tripura $(n=58)$ who live in malaria endemic area. Randomly selected individuals from the mainstream Bengali population $(n=211)$ without any history of malaria and not inhabiting in malaria endemic area were also recruited in this study.

DNA was extracted using the Chelex-100 method ${ }^{(13)}$. The extracted DNA was quantified by using NanoDrop-1000 (NanoDrop Technologies, Inc. Wilmington, DE 19810, USA). Ten autosomal STR loci, namely D3S1358, vWA, D16S539, D2S1338, D8S1179, D21S11, D18S51, D19S433, TH01 and FGA were co-amplified using AmpFlSTR ${ }^{\circledR}$ SGM Plus ${ }^{\circledR}$ PCR amplification kit (Applied Biosystems, Foster City, CA, USA). The PCR reaction was carried out in a GenAmp® PCR System 2720 (Appliled Biosystems). Thermal cycling parameters were set up according to the manufacturer's protocol.

PCR amplified fragments were separated and analyzed on ABI Prism 3100-avant Genetic Analyzer (Applied Biosystems) using POP-4 polymer and data collection software ver. 1.1. Data were sized using GeneScan Software version 3.7 and internal GeneScan-500 ROX size standard. Genotype of each locus was determined after comparison with allelic ladder using Genotyper software version 3.7 NT.

\section{Results and Discussion}

Malaria, caused by Plasmodium parasite in many parts of the world. Of the four species, Plasmodium falciparum infection is the leading cause of mortality, but the change in environment and human demography has affected the host-parasite interactions that have subsequently affected the disease spectrum. It is believed that microsatellites play a significant role in the adaptation of bacteria and perhaps higher organism to their ever changing environments. On the other hand, they have also been linked to many human diseases. ${ }^{810,12}$ It would therefore, be fascinating to explore whether the high allelic 
diversity of microsatellite region in human genome has association with a large number of infections including malaria or it may be due to population stratification.

In this study, we used a battery of microsatellite loci included in SGM Plus ${ }^{\mathrm{TM}}$ PCR amplification kit (Applied Biosystems) usually used for human identification and paternity analysis. Out of 10 microsatellite loci tested (Table 1), this study found a positive association between allele- 9 of TH01 locus in P. falciparum infected individuals and individual groups living in malaria endemic areas (Fig. 1). The TH01 locus is a tetrameric microsatellite sequence with core repeat [AATG] and present in intron 1 of thyroid peroxidase gene. The $\chi^{2}$ test applied to check the difference in allele frequency

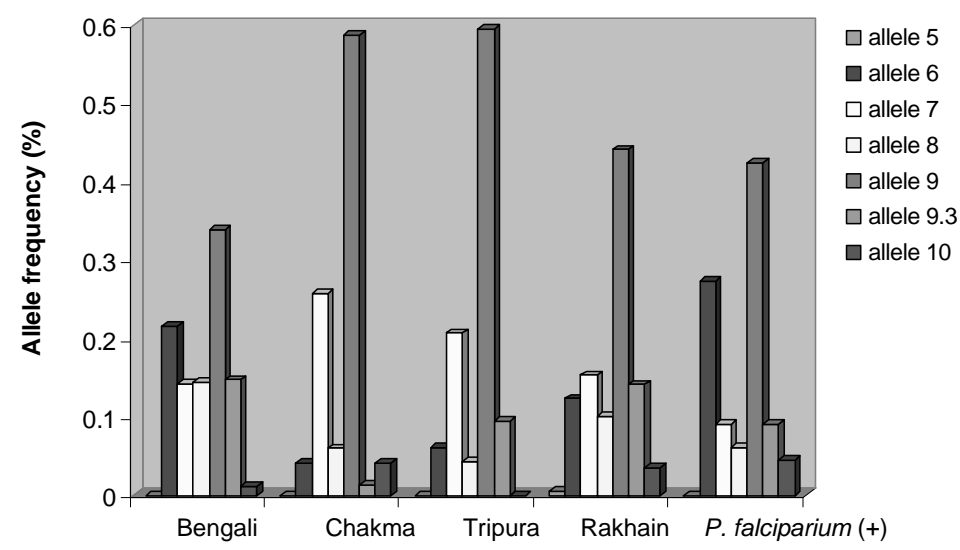

Fig 1. Allele frequency distribution at micorsatellite locus TH01 in P. falciparum positive patients and individual groups living in malaria prone and other areas of Bangladesh.

Table 1. Locus specific information of ten microsatellite markers analyzed.

\begin{tabular}{lllccl}
\hline $\begin{array}{l}\text { STR } \\
\text { locus }\end{array}$ & $\begin{array}{c}\text { Chromosomal } \\
\text { location }\end{array}$ & $\begin{array}{c}\text { Repeat } \\
\text { unit }\end{array}$ & $\begin{array}{c}\text { Allele } \\
\text { range }\end{array}$ & $\begin{array}{c}\text { No. of allele } \\
\text { observed }\end{array}$ & $\begin{array}{l}\text { Genbank } \\
\text { accession }\end{array}$ \\
\hline D3S1358 & 3p21 & [TCTA][TCTG] & $9-20$ & 20 & NT005997 \\
vWA & 12p12p-ter & [TCTA][TCTG] & $10-24$ & 28 & M25858 \\
D16S539 & 16q24q-ter & GATA & $5-15$ & 10 & G07925 \\
D2S1338 & 2q35-37.1 & [TGCC][TTCC] & $15-28$ & 14 & G08202 \\
D8S1179 & 8q24.1-24.2 & [TCTA][TCTG] & $7-19$ & 13 & G08710 \\
D21S11 & $21 q 21.1$ & [TCTA][TCTG] & $24-38$ & 70 & AP000433 \\
D18S51 & $18 q 21.3$ & AGAA & $7-27$ & 43 & L18333 \\
D19S51 & 19q21-13.1 & AAGG & $9-17.2$ & 15 & G08036 \\
TH01 & 11p15.5 & AATG & $3-14$ & 20 & D00269 \\
FGA & 4q28 & CTTT & $15-51.2$ & 69 & M64982 \\
\hline
\end{tabular}


distribution across malaria positive and other population groups showed that the estimated values were significantly higher $(p<0.01)$ in malaria positive patients and in Chakma, Tripura and Rakhain individuals living malaria endemic areas. This finding correlates with another study conducted on East Indian population by Gaikwar et al.(14) where they found higher frequency of allele- 9 in malaria positive isolates, while allele- 6 was predominant in normal groups with no clinical history of malaria.

Authors also calculated the heterozygosity of TH01 loci in all the population groups studied. Heterozygosity is a measure of the proportion of genes that are heterozygous at a particular locus and calculated by the equation $\mathrm{H}_{\mathrm{o}}=1-\sum \mathrm{pi}^{2}$ [where $\mathrm{H}_{\mathrm{o}}=$ observed heterozygosity and pi = number of homozygotes]. Fig. 2 shows that individuals living in malaria non-endemic area have a very high observed heterozygosity than individual and individuals groups living in malaria endemic areas. Heterozygosity also represents a population capacity to respond to selection and ultimate survival. The higher is the heterozygosity the better is the genetic health of a population.

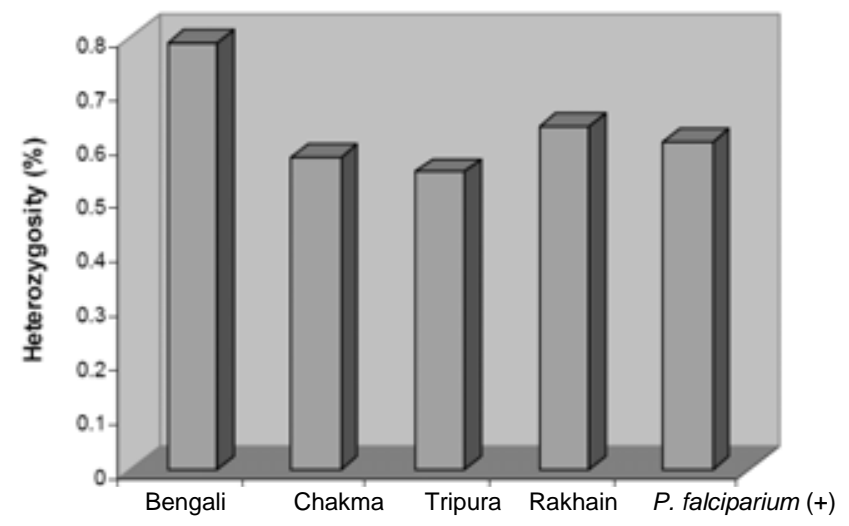

Fig. 2. Heterozygosity observed at micorsatellite locus TH01 in P. falciparum positive patients and individual groups living in the malaria prone and other areas of Bangladesh.

The Chakmas are the largest tribal minority in Bangladesh and concentrated in the central and northern part of the Chittagong Hill Tracts amidst other ethnic groups. They represent less than $1 \%$ of the total population of the country and more than $90 \%$ of them are concentrated in Rangamati and Khagrachari districts. The Tripuras are another large ethnic group in the Chittagnog Hill Tracts region. The Tripuras currently living in Bangladesh believed to have migrated from the Indian state of Tripura. At present they live in Ramgarh and Khagrachari district. The Rakhains belong to the Bhotbarmi community of Mongloids and migrated from the land Rakhain Pre, which is now Arakan in Mayanmar. More than 80 per cent of them live in Ramu, Cox's Bazar, Bandarban and Teknaf districts. As apparent from their lifestyle, they live as an isolated cluster and 
tend to have social structure in place. This ultimately push them towards inbreeding. Inbreeding is a genetic term that refers to reproduction as a result of mating between the individuals who are genetically related to each other. Inbreeding increases the chance of offspring being affected by recessive or deleterious alleles due to the lack of sufficient mixing or randomization of allele. The consequence of inbreeding is, therefore an increase in homozygosity or decease in heterozygosity. The relatively low observed heterozygosity in these ethinc groups compared to the mainstream Bengali population explains the fact (Fig. 2).

This study provided significant evidence that an individual's genotype is a product of host interaction with P. falciparum infection. The association between allele-9 (TH01) in $P$. falciparum infected individual illustrates the genetic predisposition towards the disease. Furthermore, the predominance of allele-9 at TH01 locus (AATG repeat unit) in the individuals inhabiting in malaria prone area suggests that the genetic predisposition towards malaria is an archaic phenomena.

\section{References}

1. Lander ES, LM Linton, B Birren, C Nusbaum, MC Zody, J Baldwin, et al. 2001. International Human Genome Sequencing Consortium. Initial sequencing and analysis of human genome. Nature 409: 860-921.

2. Weber JL 1990. Informativeness of human (dC-dA)n.(dG-dT)n polymorphism. Genomics 7: 524-530.

3. Gill P, PI Ivanov, C Kimpton, R Piercy, N Benson, G Tully, I Evett, E Hagelberg and K Sullivan 1994. Identification of the remains of the Romanov family by DNA analysis. Nat. Genet. 6: 130-135.

4. Butler D, J Maurice and C O'Brein 1997. Time to put malaria control on global agenda. Nature 386: 535-536.

5. Modiano D, V Petrarca, BS Sirima 1996. Different response to Plasmodium falciparum malaria in west African sympatric ethnic groups. Proc. Natl. Acad. Sci. USA. 93: 13206-13211.

6. Abel L, M Cot, L Mulder, P Carnevale and J Feingold 1992. Segregation analysis detects a major gene controlling blood infection level in human malaria. Am. H. Hum. Genet. 50: 1308-1317.

7. Sjoberg K, JP Lepers, L Raharimlalala 1992. Genetic regulation of human anti-malarial antibodies in twins. Proc. Natl. Acad. Sci. USA. 89: 2101-2104.

8. Pasvol G, DJ Weatherall and RJ Wilson 1978. Cellular mechanism for the protective effect of hemoglobin $S$ against $P$. falciparum malaria. Nature 274: 701-703.

9. Hill AV, CE Allsopp, D Kwiatkowski 1991. Common west African HLA antigens are associated with protection from severe malaria. 352: 595-600.

10. TN Williams, K Maitland, S Bennet 1996. High incidence of malaria in alpha-thassemic children. Nature 383: 522-525.

11. C Ruwende, SC Khoo, RW Snow 1995. Natural selection of hemi- and heterozygotes for G6PD deficiency in Africa by resistance to severe malaria. Nature 376: 246-249. 
12. ER Maxon and C Wills 1999. DNA microsatellites: agents of evolution? Sci. Am. 280: 94-99.

13. Walsh PS, DA Metzger and R Higuchi 1991. Chelex 100 as a simple medium for simple extraction of DNA for PCR-based typing from forensic material. Biotechniques 10: 413506.

14. Gaikwad S, R Asma, N Kumar, R Trivedi and VK Kashyap. 2005. Host microsatellite alleles in malaria predisposition? Malaria Journal. 4(50): 1-5.

(Manuscript received on 18 March, 2010; revised on 19 April, 2010) 